\title{
Estudo G.R.I.P.E. (Garantia de Receituário Incentivado pelo Especialista)
}

António Macedo, ${ }^{*}$ Elias Filipe, ${ }^{* *}$ Elisa Guerra, ${ }^{* * *}$ Paula Pamplona, ${ }^{* * * *}$ Sónia Cardoso, ${ }^{* * * * *}$

\section{RESUMO}

Introdução: A vacinação anual é o principal método de prevenção e controlo da infecção gripal sazonal e das suas complicações, devendo ser dirigida aos grupos populacionais de alto risco, como os idosos (idade $\geq 65$ anos). Os médicos de família (MF) têm um papel importante no controlo desta doença no sentido de promover a vacinação.

Objectivo: Avaliar e garantir a qualidade do aconselhamento e/ou prescrição da vacina anti-gripal pelos MF nos idosos.

Tipo de estudo: Descritivo e transversal.

Local: Cinco centros de saúde (CS) da Região Norte.

População: Todos os idosos inscritos nos CS estudados.

Métodos: Dimensão estudada: qualidade técnico-científica. Unidade de estudo: 375 utentes inscritos nas listas de MF dos CS avaliados, que completaram 65 anos até 30 de Setembro de 2006. Foram avaliados cinco médicos de família em cada CS no período de 1 de Setembro de 2006 e 28 de Fevereiro de 2007. Base de estudo: amostra aleatória sistemática de 375 utentes, de base institucional. Tipo de dados: de processo. Fonte de dados: processos clínicos e SAM. Tipo de avaliação: interna. Critérios: Registo do aconselhamento ou prescrição da vacina anti-gripal no período em estudo aos utentes idosos. Avaliação temporal: retrospectiva. Tipo de intervenção prevista: estrutural e educacional.

Resultados: Em 49,3\% dos casos não foi encontrado nenhum registo de aconselhamento e/ou prescrição da vacina da gripe no grupo alvo. Em $50,7 \%$ dos processos a vacina tinha sido aconselhada $(5,1 \%)$ ou prescrita $(45,6 \%)$, permitindo alcançar o padrão de qualidade suficiente. Entre as diferentes unidades de saúde a taxa de aconselhamento/prescrição variou entre 36\% (insuficiente) e $68 \%$ (suficiente).

Conclusões: É necessária uma melhoria contínua da qualidade da prescrição da vacina da gripe nos grupos de risco. Propõe-se a formação de um grupo de trabalho para o Desenvolvimento Contínuo da Qualidade nesta área em cada Centro de Saúde.

Palavras-Chave: Gripe; Vacinação; Garantia da Qualidade dos Cuidados de Saúde.

\section{INTRODUÇÃO}

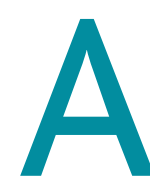

gripe é uma infecção aguda das vias respiratórias causada pelo vírus Influenza, altamente contagiosa e que se apresenta, na maior parte das vezes, de forma epidémica.

Afecta anualmente milhões de pessoas em todo o mundo, com uma taxa de ataque anual estimada de 5 $-10 \%$ nos adultos e $20-30 \%$ nas crianças. ${ }^{1}$ A gripe e a pneumonia são a sexta causa principal de morte e a

\footnotetext{
* Interno de Medicina Geral e Familiar no C.S. Senhora da Hora

**Interno de Medicina Geral e Familiar no C.S. Paredes/Rebordosa

*** Interna de Medicina Geral e Familiar no C.S. S. Mamede de Infesta

****Interna de Medicina Geral e Familiar no C.S. Maia/Águas Santas

*****Interna de Medicina Geral e Familiar no C.S. Rio Tinto/S. Pedro da Cova (Brás-Oleiro)
}

quinta causa principal de morte nos adultos com idade igual ou superior a 65 anos, nos Estados Unidos. ${ }^{2}$ Em Portugal, entre 2000 e 2004, ocorreram 152.731 internamentos em hospitais públicos, com diagnóstico de pneumonia e gripe, dos quais $63 \%$ reportaram-se a pessoas com idade superior a 65 anos, originando uma taxa anual média bruta de internamentos de $1.179,4$ por $10^{5}$ habitantes. No mesmo período ocorreram 16.182 óbitos, por pneumonia e gripe, no grupo etário referido, correspondendo a $91 \%$ do total de mortes registadas por esta causa. ${ }^{3}$

A propagação da gripe numa comunidade resulta habitualmente num aumento do absentismo laboral, das hospitalizações motivadas por doenças respiratórias e das mortes imputadas directamente à infecção 
gripal e suas complicações. ${ }^{4-6}$ Como em quase todas as doenças preveníveis, resulta também em sofrimento pessoal e familiar e no agravamento e agudização de doenças crónicas (nomeadamente cardiovasculares, muito mais frequentes nos grupos etários acima dos 65 anos). Tudo isto representa um grave problema de saúde pública pela sobrecarga considerável em termos de custos sociais e de cuidados de saúde.

A gripe atinge todos os grupos etários e apesar de ser relativamente benigna, dado que evolui normalmente para a cura, pode provocar graves complicações em indivíduos mais susceptíveis ou mais debilitados. A taxa de mortalidade por gripe começa a aumentar na idade média da vida, sendo mais elevada nos indivíduos com idades superiores a 65 anos e/ou com patologia crónica subjacente. Este grupo etário apresenta taxas de hospitalização e de mortalidade por pneumonia e gripe superiores às da população em geral, correspondendo-lhes cerca de $90 \%$ ou mais das mortes por gripe. ${ }^{7}$

A vacinação anual é o principal método de prevenção e controlo da infecção gripal sazonal, bem como das suas complicações graves, devendo ser dirigida essencialmente aos grupos populacionais de alto risco, como no caso dos idosos, doentes portadores de doenças respiratórias, cardíacas crónicas e diabéticos, bem como profissionais de saúde que têm contacto com esses grupos. A vacinação é segura e eficaz evitando o aparecimento da gripe em até $75 \%$ das situações e diminuindo a gravidade da doença em $98 \%$ dos casos. ${ }^{1,8}$

A vacinação da população idosa não institucionalizada pode reduzir o número de internamentos em 25 a $39 \%$ e a mortalidade global em 39 a $75 \%$ durante a época gripal. ${ }^{1,7}$ Nos idosos institucionalizados a vacina contra a gripe pode reduzir os internamentos (por todas as causas) em $50 \%$, o risco de pneumonia em cerca de $60 \%$ e o risco de morte (por todas as causas) em $68 \% .{ }^{1}$ Por estes motivos, o aumento da cobertura vacinal neste grupo reveste-se de crucial importância. ${ }^{8}$

O Centers for Disease Control and Prevention (CDC) estima que, para cada milhão de indivíduos de alto risco vacinados, são prevenidas cerca de 900 mortes e 1.300 hospitalizações durante uma época gripal. ${ }^{9-12} \mathrm{~A}$ Direcção-Geral da Saúde (DGS) aconselha a vacinação, preferencialmente no Outono, das pessoas consideradas com alto risco de desenvolver complicações pós-infecção gripal, entre os quais se incluem os indivíduos com 65 ou mais anos de idade, particularmente os residentes em lares ou outras instituições. ${ }^{13}$

De acordo com os objectivos da OMS para 2006, considerou-se prioritário aumentar a cobertura vacinal nas pessoas com idade igual ou superior a 65 anos para $50 \% .{ }^{13}$ Nas três últimas épocas gripais a estimativa da proporção de indivíduos com idade superior a 65 anos que declararam estar vacinados contra a gripe foi de $39,0 \%$ na época gripal de $2004 / 2005$, de $41,6 \%$ na de 2005/2006 e de 50,4\% na de 2006/2007..$^{14}$ Considerando o número de doses comercializadas em Portugal nos últimos anos, estima-se uma cobertura vacinal contra a gripe da ordem dos $15 \%$ da população em geral. ${ }^{14}$

A OMS pretende que a vacina contra a gripe atinja, até 2010, uma cobertura de $75 \%$ da população acima dos 65 anos. ${ }^{1}$ Os profissionais de saúde têm um papel muito importante no controlo desta doença, particularmente na época do Outono/Inverno, no sentido de promover a vacinação essencialmente nos grupos de risco.

É objectivo deste estudo avaliar e garantir a qualidade do aconselhamento e/ ou prescrição da vacina antigripal pelos médicos de família (MF) nos utentes com idade igual ou superior a 65 anos.

\section{MÉTODOS}

A dimensão estudada foi a qualidade técnico-científica do aconselhamento e/ou prescrição da vacina da gripe aos utentes com idade igual ou superior a 65 anos de idade.

A unidade de estudo envolveu os utentes inscritos nas listas de cinco Médicos de Família (MF) de cada um dos Centros de Saúde (CS) onde os autores do estudo exercem a sua actividade (CS Senhora da Hora, CS Paredes/Rebordosa, CS S. Mamede de Infesta, CS Maia/Águas Santas (Sede) e CS Rio Tinto/S. Pedro da Cova-Extensão de Saúde de Brás-Oleiro), que completaram 65 anos até 30 de Setembro de 2006. Os profissionais envolvidos na avaliação foram cinco MF de cada unidade de saúde avaliada seleccionados por conveniência ao permitirem o estudo da sua lista de utentes (no total de 25 médicos). O tempo em estudo foi dirigido à época gripal 2006/2007 (definido pelos autores como o período compreendido entre 1 de Setembro de 2006 e 28 de Fevereiro de 2007).

O tipo de dados analisados foi de processo.

A fonte de dados envolveu os processos clínicos in- 
QUADRO I. Critério avaliado e padrão de qualidade adoptado

\section{Critério}

Padrão

Registo do aconselhamento

e/ou prescrição da vacina

anti-gripal no período de

1 de Setembro de 2006 a

28 de Fevereiro de 2007 em

utentes com idade $\geq 65$ anos

formatizados, nomeadamente a versão 7.1 do Sistema de Apoio ao Médico (SAM ${ }^{\circledast}$ ) pesquisando o campo de prescrições anteriores no período de tempo em estudo bem como os processos clínicos manuscritos, isto é, as Fichas Clínicas Individuais - Adultos (ARSN-Mod. 121.01 e ULS-Mod.121.01) ou Folhas de Consulta Adultos (ARSN-13/99.017 e ULS Mod.13/99.017)).

O tipo de avaliação foi interna.

O critério avaliado e o padrão de qualidade adoptado encontram-se descritos no Quadro I. O padrão utilizado foi decidido entre os autores com base nas metas propostas para 2006/2007 pela DGS. ${ }^{11}$

A colheita de dados foi efectuada pelos autores entre 22 de Outubro e 23 de Novembro de 2007.

A avaliação foi retrospectiva.

A amostra foi de base institucional. Foram seleccionados 15 utentes da lista de cada MF, a partir do programa informático SINUS ${ }^{\circledast}$ (inscritos no CS em estudo e organizados por ordem alfabética), de forma aleatória sistemática (de 10 em 10).

O tipo de intervenção prevista é de base educacional e estrutural.

\section{RESULTADOS}

Em cada unidade de saúde foram analisados 75 processos clínicos que no total perfizeram 375 casos avaliados. Em 185 casos (49,3\%) não foi encontrado nenhum registo de prescrição ou aconselhamento da vacina da gripe no grupo alvo. No entanto, em 190 processos $(50,7 \%)$ a vacina tinha sido aconselhada $(5,1 \%)$ ou prescrita (45,6\%), permitindo alcançar, na globalidade, o padrão de qualidade suficiente (Quadro II).

As taxas de não prescrição, aconselhamento e prescrição da vacina da gripe por unidade de saúde analisada é detalhada no Quadro II.

Na comparação entre as diferentes unidades de saúde pode-se verificar que o padrão máximo de qualidade não foi atingido em nenhuma. Consideraram-se suficientes os resultados obtidos no CS Paredes/Rebordosa, que obteve o melhor resultado (68\%), e CS Maia/ /Águas Santas (Figura 1).

Por seu lado, os resultados dos centros de saúde da Senhora da Hora e S. Mamede de Infesta e a unidade de saúde de Brás-Oleiro não atingiram o padrão mínimo de qualidade, apesar destas duas últimas unidades estarem perto de o atingir (ambas obtiveram 49,3\% de aconselhamento/prescrição da vacina da gripe). O CS da Senhora da Hora registou o pior resultado com $36 \%$ de taxa de registo de aconselhamento e/ou prescrição. Assim sendo, estas três unidades de saúde apresentaram resultados insuficientes.

\section{DISCUSSÃO}

De acordo com os resultados obtidos verifica-se que, apesar do padrão total de qualidade ter sido suficiente com uma taxa de aconselhamento/prescrição da vacina da gripe de $50,7 \%$, a taxa efectiva de prescrição des-

QUADRO II. Prescrição da vacina da gripe nas Unidades de Saúde avaliadas

\begin{tabular}{|l|c|c|c|c}
\multirow{2}{*}{ Unidade de Saúde } & \multicolumn{3}{|c|}{ Prescrição da Vacina da Gripe } & \multirow{2}{*}{ Processos Avaliados } \\
\cline { 2 - 5 } & Não Prescrita & Aconselhada & Prescrita & $75(100)$ \\
\hline Senhora da Hora (\%) & $48(64,0)$ & $1(1,3)$ & $26(34,7)$ & $75(100)$ \\
\hline Paredes/Rebordosa (\%) & $24(32,0)$ & $8(10,7)$ & $43(57,3)$ & $75(100)$ \\
\hline S. Mamede de Infesta (\%) & $38(50,7)$ & $4(5,3)$ & $33(44,0)$ & $75(100)$ \\
\hline Maia/Águas Santas (\%) & $37(49,3)$ & $3(4,0)$ & $35(46,7)$ & $75(100)$ \\
\hline Brás-Oleiro (\%) & $38(50,7)$ & $3(4,0)$ & $34(45,3)$ & $375(100)$ \\
\hline Total (\%) & $185(49,3)$ & $19(5,1)$ & $171(45,6)$ & \\
\hline
\end{tabular}




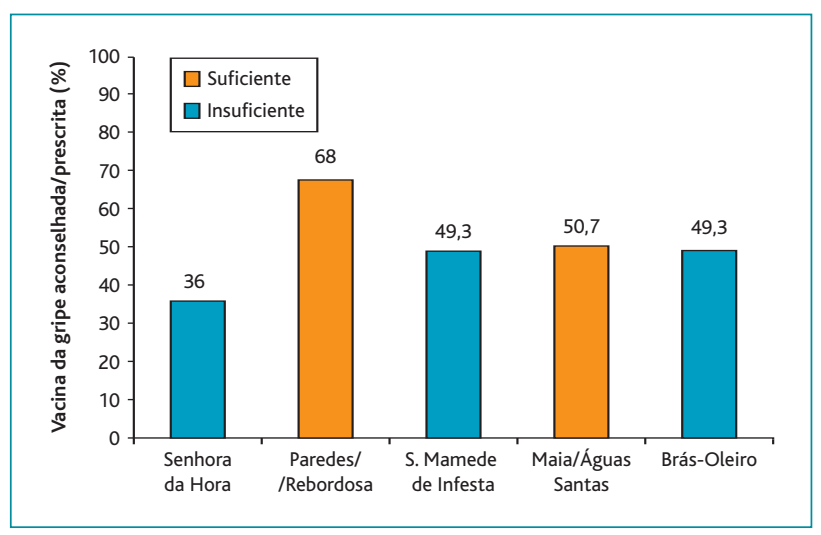

Figura 1. Taxa de aconselhamento/prescrição da vacina da gripe nas Unidade de Saúde avaliadas

ta vacina foi de 45,6\%. Para 2006 a Organização Mundial de Saúde (OMS) considerou prioritário aumentar a cobertura vacinal nas pessoas com idade igual ou superior a 65 anos para 50\%. Deste modo, apesar da qualidade técnico-científica dos médicos de família ter sido suficiente (de acordo com o padrão de qualidade adoptado), torna-se evidente que existe ainda algum caminho a percorrer no sentido de melhorar a taxa de prescrição e consequentemente a cobertura vacinal. Só assim se conseguirá atingir o objectivo proposto pela OMS que pretende aumentar até $75 \%$ a cobertura vacinal em 2010. ${ }^{1}$ Salienta-se que a colheita de dados para este estudo foi realizada unicamente através da consulta de processos clínicos tornando a qualidade dos dados obtidos dependente dos registos de cada médico de família. Nalguns casos a prescrição da vacina contra o vírus influenza foi efectuada de forma manual, o que torna o registo deste acto ainda mais importante para efeitos de estudos de avaliação da qualidade. Assim, supõe-se que, nalguns casos, se possa verificar uma ausência de registos conduzindo necessariamente a uma subvalorização dos resultados obtidos. É de salientar que o presente estudo, por alguns constrangimentos extrínsecos aos investigadores, apresenta uma debilidade na técnica de amostragem, nomeadamente a escolha por conveniência de 5 médicos para representarem a totalidade de uma unidade de saúde bem como o estudo de apenas 15 doentes para representarem a totalidade da lista de um médico. Os investigadores reconhecem esta falência e sugerem, numa próxima avalia- ção, a utilização de outras técnicas de amostragem para obter uma melhor representatividade da população em estudo.

Entre as diferentes unidades de saúde é evidente a existência de resultados díspares, com a taxa de aconselhamento/prescrição a variar entre 36 e $68 \%$ e com apenas duas unidades de saúde a atingirem o padrão de qualidade suficiente. Este facto pode justificar-se através da constatação de que a abordagem para este acto médico foi diferente entre unidades. Numas unidades optou-se por uma abordagem de conveniência em que era prescrita a vacina se o utente do grupo alvo tivesse uma consulta com o seu médico de família durante o início da época gripal, enquanto noutras se optou pela elaboração de listas dos utentes a vacinar no início da época gripal que serviram para controlar a quem era prescrita a vacina, sendo os utentes convocados por escrito. $\mathrm{O}$ presente estudo não pretendeu avaliar a diferença entre estas atitudes mas seria interessante verificar a sua existência com a realização de outros estudos nesta área.

Embora não fazendo parte do objectivo deste estudo, verificou-se a existência, durante a época gripal, de consultas dirigidas aos utentes do grupo alvo em que não foi aproveitada a oportunidade para a prescrição da vacina. O esquecimento por parte do médico e a falta de interesse na procura da vacina por parte do utente podem contribuir para que tal aconteça, impondo-se assim a necessidade de investir em meios de aviso de prescrição para os médicos e actividades de educação para a saúde dos utentes para colmatar esta falha.

Apesar dos resultados globais suficientes no contexto actual, num futuro próximo ficarão muito aquém das expectativas, tornando-se necessária uma melhoria contínua da qualidade da prescrição da vacina da gripe neste grupo de risco. Este estudo tem por objectivo a sensibilização da classe médica no sentido de fomentar uma mudança de atitude que assegure a realização de tarefas clínicas com qualidade e com benefícios evidentes para a saúde da população.

Pretende-se criar em cada centro de saúde um grupo de trabalho constituído por médicos, enfermeiros e administrativos que terá a seu cargo a elaboração de um Programa Para o Desenvolvimento Contínuo da Garantia da Qualidade da Vacinação da vacina anti-gripal. Este grupo organizará acções de formação dirigidas às 
equipas de saúde familiar com vista a apresentar este estudo e sensibilizar para a problemática da qualidade de prescrição da vacina da gripe nos grupos de risco.

Este grupo também ficaria responsável pela elaboração de um protocolo interno com as seguintes orientações:

1. Alertar os profissionais de saúde para esta condição sazonal e medidas de prevenção/actuação, onde se inclui a importância da vacinação;

2. Criar um sistema de alerta informático que permita aos médicos de família uma rápida identificação dos utentes a convocar para a prescrição da vacina dentro do período determinado;

3. Elaborar, no início da época gripal, uma listagem, através do programa SINUS ${ }^{\circledR}$, dos utentes com idade igual ou superior a 65 anos de idade, a distribuir por cada médico de família de forma a controlar a prescrição da vacina da gripe neste grupo de risco;

4. Convocar em Dezembro, através de listagem do SINUS, os utentes com mais de 65 anos aos quais não tenha ainda sido prescrita a vacina numa primeira fase;

5. Motivar todos os grupos profissionais (médicos, enfermeiros e administrativos) a realizar um aconselhamento oportunista da vacina anti-gripal aos utentes que recorram ao centro de saúde por outros motivos (como é o caso dos pedidos de medicação crónica, realização de cuidados de enfermagem, entre outros);

6. Adoptar estratégias inter-pares que visem colmatar deficiências no aconselhamento da vacina da gripe como actualização de Normas Directivas e problemas inerentes ao doente (problemas económicos, sociais, culturais, religiosos e ambientais).

Outro grupo de medidas estruturais a aplicar poderá passar pela criação de incentivos de ordem profissional a todos os envolvidos na aplicação do protocolo pelo atingimento dos objectivos propostos no documento.

O mesmo grupo de trabalho poderá ficar responsável pelo desenvolvimento de acções de sensibilização/educação para a saúde dirigidas a este grupo de risco e profissionais de saúde, através da realização de palestras, distribuição de panfletos e afixação de posters com a finalidade de aumentar a percepção dos riscos da infecção gripal, a eficácia da vacina e combater o

$\begin{aligned} & \text { QUADRO III. Cronograma proposto para o desenrolar } \\
& \text { deste Programa para o Desenvolvimento Contínuo da } \\
& \text { Garantia da Qualidade da Vacinação }\end{aligned}$
\begin{tabular}{ll} 
Período & Actividades \\
\hline Até Março de 2008 & $\begin{array}{l}\text { Criação de um grupo de trabalho } \\
\text { para o desenvolvimento } \\
\text { contínuo da qualidade em } \\
\text { cada unidade de saúde }\end{array}$ \\
\hline Época gripal 2008/2009 & $\begin{array}{l}\text { Aplicação das medidas } \\
\text { correctoras propostas }\end{array}$ \\
\hline Abril de 2009 & Nova Avaliação da Qualidade
\end{tabular}

medo e preconceitos sobre os efeitos adversos da vacinação.

À medida que o processo for decorrendo o grupo de trabalho investigará outros problemas relativos à prescrição e prestação de cuidados neste grupo de risco com vista a uma melhoria contínua da qualidade. Para terminar, na sequência da avaliação do aconselhamento e/ou prescrição da vacina anti-gripal, seria oportuno avaliar a taxa de cobertura efectiva da vacinação no sentido de identificar falhas e facilitar a implementação de medidas facilitadoras do atingimento das metas propostas pela OMS.

Segue-se um cronograma proposto para o desenrolar deste Programa Para o Desenvolvimento Contínuo da Garantia da Qualidade da Vacinação (Quadro III).

\section{REFERÊNCIAS BIBLIOGRÁFICAS}

1. World Health Organization. Weekly epidemiological record (Relevé épidémiologique hebdomaire).WHO. 19 August 2005, 80th Year (19 Août 2005, 80e Année); vol. 80 (33): 277-88. Disponível em: http://www. who.int/wer/2005/wer8033.pdf [acedido em 10/10/2007].

2. Thompson WW, Shay DK, Weintraub E, Brammer L, Cox N, Anderson L, et al. Mortality associated with influenza and respiratory syncytial virus in the United States. JAMA 2003 Jan; 289 (2): 179-86.

3. Nicolau R, Machado A, Falcão JM, Nunes B. Pneumonia e gripe: Análise da mortalidade e dos internamentos hospitalares da população idosa por concelho de residência. ONSA Observações. 2007; 37 (Novembro 2007). Disponível em: http://www.onsa.pt/conteu/pub_observacoes-037_onsa.pdf [acedido em 8/12/2008].

4. Bridges CB, Fukuda K, Uyeri TM, Cox NJ, Singleton JA; Centers for Disease Control and Prevention, Advisory Committee on Immunization Practices. Prevention and control of influenza: recommendations of the Advisory Committee on Immunization Practices. MMWR Recomm Rep 2002 Apr 12; 51 (RR-3): 1-31.

5. Lui KJ, Kendal AP. Impact of influenza epidemics on mortality in the 
United States from October 1972 to May 1985. Am J Public Health 1987 Jun; 77: 712-6.

6. Glezen WP. Serious morbidity and mortality associated with influenza epidemics. Epidemiol Rev 1982; 4: 25-44.

7. Thompson WW, Shay DK, Weintraub E, Brammer L, Bridges CB, Cox NJ, et al. Influenza-associated hospitalizations in the United States. JAMA 2004 Sep 15; 292 (11): 1333-40.

8. Nichol KL, Nordin JD, Nelson DB, Mullooly JP, Hak E. Effectiveness of influenza vaccine in the community-dwelling elderly. N Engl J Med 2007 Oct 4; 357 (14): 1373-81.

9. Nichol KL, Nordin J, Mullooly J, Lask R, Fillbrandt K, Iwane M. Influenza vaccination and reduction in hospitalizations for cardiac disease and stroke among the elderly. N Engl J Med 2003 Apr 3; 348 (14):1322-32.

10. Nordin J, Mullooly J, Poblete S, Strikas R, Petrucci R, Wei F, et al. Influenza vaccine effectiveness in preventing hospitalizations and deaths in persons 65 years or older in Minnesota, New York and Oregon: data from 3 health plans. J Infect Dis 2001 Sep 15; 184 (6): 665-70.

11. Bridges CB, Thompson WW, Meltzer MI, Reeve GR, Talamonti WJ, Cox $\mathrm{NJ}$, et al. Effectiveness and cost-benefit of influenza vaccination of healthy working adults: a randomized controlled trial. JAMA 2000 Oct 4; 284 (13): 1655-63.
12. Gross PA, Hermogenes AW, Sacks HS, Lau J, Levandowski RA. The efficacy of influenza vaccine in elderly persons: a meta-analysis and review of the literature. Ann Intern Med 1995 Oct 1; 123 (7): 518-27.

13. Direcção Geral de Saúde. Circular informativa. Assunto: Gripe: Vacinação Contra a Gripe em 2006/2007, n 40/DIR/G, de 21/09/06.

14. Direcção Geral de Saúde. Circular informativa. Assunto:Vacinação Contra a Gripe Sazonal em 2007/2008, n 35/DSCS/DPCD, de 26/09/07.

\section{AGRADECIMENTOS}

Os autores desejam agradecer à Dra. Ana Sardinha e à Dra. Emília Teixeira pelos conhecimentos e apoio prestados durante a realização deste estudo.

\author{
ENDEREÇO PARA CORRESPONDÊNCIA \\ António Alberto Gonçalves Macedo \\ Rua D. João de Castro, 225 \\ 4435-674 Baguim do Monte \\ E-mail: antmac.antonio@gmail.com \\ Tlm: 912883206
}

Recebido em 28/04/08

Aceite para publicação em 14/01/09

\section{ABSTRACT}

Introduction: Annual vaccination is the main method of prevention and control of the influenza seasonal infection and its complications. It must be directed to the population groups of high risk, like the elderly (age equal or superior to 65 years). The family doctor has an important role in controlling this disease by promoting vaccination.

Aim: Evaluate and guarantee the quality of the advising and/or prescription of the anti-influenza vaccine by the Family Doctor (FD) in elderly patients.

Type of study: Descriptive and transversal.

Place: 5 Primary Care Centers (PCC) on the Northern Region of Portugal.

Population: All elderly registered on the PCC studied.

Methodology: Studied dimension: technical-scientific quality. Unity of study: 375 patients registered in the lists of FD of the evaluated PCC, who completed 65 years up to 30 of September of 2006. Five FD were evaluated at each PCC between September of 2006 and February of 2007. Study Base: random systematic sample of 375 patients, of institutional base. Type of data: process. Data source: Medical files (paper and digital). Type of evaluation: internal. Criteria: Registry of advising or prescription of the anti-influenza vaccine in the period of study on elderly patients. Temporal evaluation: retrospective. Type of predicted intervention: structural and educational.

Results: In $49.3 \%$ of the cases there was not found any register of advising and/or prescription of the influenza vaccine. In $50.7 \%$ the vaccine has been advised (5.1\%) or prescribed (45.6\%), allowing to reach the standard of sufficient quality. Between the different unities of health the percentage of advising/prescription varied between $36 \%$ (insufficient) and $68 \%$ (sufficient).

Discussion: There is need for a continuous quality improvement of the influenza vaccine prescription in the risk groups. We proposed the formation of a work group to the Continuous Quality Development in this area in each PCC.

Keywords: Influenza; Influenza Vaccines; Vaccination; Health Care Quality Assurance. 